\title{
ASSESSMENT OF WATER QUALITY OF BHAGIRATHI FROM GANGOTRI TO RISHIKESH USING RS AND GIS TECHNIQUES
}

\author{
Rajesh Arora ${ }^{1, *}$, Harish Chandra Joshi ${ }^{2}$, I.P.Pandey ${ }^{3}$ and V.K.Tewari ${ }^{4}$ \\ 1, ${ }^{*}$ Department of Chemistry, Graphic Era University, Dehradun- 248001, Uttarakhand, India \\ ${ }^{2}$ Departmentof Chemistry, Uttaranchal University, Dehradun-248007, Uttarakhand, India \\ ${ }^{3}$ Departmentof Chemistry, DAV (PG) College, Dehradun-248001, Uttarakhand, India \\ ${ }^{4}$ Department of Mechanical Engineering, Graphic Era University, Dehradun- 248001, \\ Uttarakhand, India \\ *E-mail: cars1425202@gmail.com
}

\begin{abstract}
Bhagirathi river is the tributary river of Ganges. Pollution is commonly regarded as the resulted of the industrial revolution. By increasing, industrial activity environmental quality of the area deteriorates. It is necessary to know details about the different physicochemical parameters and water samples were analyzed for various heavy metals like nickel, copper, lead, cadmium, chromium, iron, zinc, manganese, magnesium, and arsenic used for testing of water quality and the special concern is because they produce in water chronic poisoning in aquatic animals. For the development planning of the river two main tools, Remote Sensing System and Geographic Information Systems were used in the present study.
\end{abstract}

Keywords: GIS and RS, Water Quality Index (WQI), Physico-chemical analysis, Heavy metals, Bhagirathi river.

(C) RASĀYAN. All rights reserved

\section{INTRODUCTION}

Bhagirathi river is the tributary river of the Ganges in Garhwal Himalayas of Uttarakhand Bhagirathi river is originating from the Gaumukh in Gangotri glacier at an altitude of $3892 \mathrm{msl}$ and passes via thickly populated towns like Gangotri, Harshil, Uttarkashi, Tehri, and Rishikesh. River Bhagirathi and River Alaknanda meet at Devprayag flow in the name of Ganges ${ }^{1,2}$. Analysis of major ion chemistry of the Ganga source water - the Bhagirathi, the Alaknanda and their tributaries to assess the chemical weathering processes in the high altitude Himalayas, indicated $\mathrm{Ca}^{+2}, \mathrm{Mg}^{+2}, \mathrm{HCO}_{3}{ }^{-1}$ and $\mathrm{SO}_{4}^{-2}$ were among the most abundant ions in these rivers ${ }^{3}$. The rapid increase in world population coupled with increasing food demand over past few decades has exerted enormous pressures on the inventory of natural resources based on earth ${ }^{4}$. This has resulted in challenging long-term sustainability of ecological balance and environmental well-being. The rapid all-round developmental activities worldwide call for a holistic approach in identifying parameters affecting the environment and enlisting the mitigating measures ${ }^{5}$.The adverse impact of the over the use of surface and ground waters on the environment is required to be minimized.The physicochemical parameters of Bhagirathi river in the Tehri dam reservoir was analyzed during the September 2007 and February 2008 in the program of India - Ibadan ${ }^{6}$. The physicochemical parameters were analyzed using standard methods ${ }^{7-10}$ for water analysis. The following parameter value ranges were obtained: water temperature $16.5-29.3^{\circ} \mathrm{C}$, transparency $55-158 \mathrm{~cm}$, turbidity $1-12 \mathrm{NTU}, \mathrm{pH}$ 4-9.8, dissolved oxygen $6.9-11.75 \mathrm{mg} / \mathrm{l}$, conductivity $59.6-93.5 \mathrm{mg} / \mathrm{l}$, total dissolved solid 31.04- 46.8 $\mathrm{mg} / \mathrm{l}$, alkalinity $40-78.2 \mathrm{mg} / \mathrm{l}, \mathrm{Ca}^{2+} 7.9-17.6 \mathrm{mg} / \mathrm{l}$, the quality of water influences the health status of human beings; hence analysis of water for properties like chemical, physical and biological properties including trace elements contents are very important for public health studies ${ }^{11}$.A lot of heavy metals, sewage effluents, and their compounds are released continuously in the Rivers because of the rapid industrialization and urbanization. Due to the release of the pollutants like zinc and iron, it causes 
paralysis, sterility, poliomyelitis, filariasis, cancer, meningitis in animals. The enlargement of oxygenconsuming decomposers, mainly bacteria and fungi are encouraged, when organic matter is added to the surface water. As bacteria and fungi consume oxygen so that they reduce the oxygen supply and thus the aquatic species such as fish and shell fish have perished.The success of planning for developmental activities depends on the quality and quantity of information available on both natural and socioeconomic resources ${ }^{12}$.It is therefore essential to devise the ways and means of organizing computerized information systems.Remote sensing technology (RS) and Geographic information system (GIS) are the latest tools available to store retrieve and analyze different types of data for management of natural resources. GIS facilitates systematic handling of data to generate information in a devised format. One of the greatest advantages of using remote sensing data for hydrological modeling and supervise its ability to generate informational spatial and temporal domain, which is very pivotal for successful model analysis, prediction, and validation.

\section{Study Area and Collection of the Sample}

\section{EXPERIMENTAL}

The study period is divided into three seasons mainly summer (March-June), winter (Nov-Feb) and Monsoon (July- Oct).The water is collected from the different five locations that are:Gangotri, Harshil, Uttarkashi, Tehri, and Rishikesh in three consecutive years 2013- 2016. The samples were taken into plastic jerry canes and brought to the laboratory with precautions and all samples were labeled properly.

\section{Analysis of Physico-chemical Property and Heavy Metals}

Standard analytical methods (APHA 1990, 1995,2009, Trivedi and Goel, 1986 etc.) All the parameters like $\mathrm{pH}$, temperature, acidity, carbon dioxide, colour, odour, taste, turbidity, TDS, TSS, hardness, chloride, sulphates, phosphates, DO, COD, BOD, reactive silica, sulphate, hydrogen sulphide, total alkalinity, carbonates, bicarbonates, total phosphorus, magnesium and heavy metals like $\mathrm{Fe}, \mathrm{Zn}, \mathrm{Co}, \mathrm{Cu}$, $\mathrm{As}, \mathrm{Pb}, \mathrm{K}, \mathrm{Cd}$, Ni etc. will be determined as per standard methods.

\section{RESULTS AND DISCUSSION}

From the observations water in the temperature range of $7^{\circ} \mathrm{C}$ to $11^{0} \mathrm{C}$ (see point 29 in Tables 1-15) has a pleasant taste and is refreshing. At higher temperature with less dissolved gases, the water becomes tasteless and even does not quench the thirst. The average temperature of the sample decreases from summer to winter session in three consecutive years (2013-2016) in all five sample stations however the temperature of all five samples continuously increases in the summer season from Gangotri to Rishikesh (19.43 to 20.77) this is due to the atmosphere and higher heating conditions of weather. Sample station of Tehri and Rishikesh, river water temperature is higher $\left(20.77{ }^{\circ} \mathrm{C}\right.$ ) (see point 2 in Table-4,5,9,10,14,15). The observed value of temperature shows a slight increase in temperature as we move down the river and also changed the taste of river water.But the trend is not always regular as some places the width of the river increases or decreases due to which suggested that the surface having larger area resulting in greater evaporation this causing cooling effect ${ }^{13}$. The observed temperature value from Gangotri to Harshil is low from summer to winter which is ideal for drinking and aquatic life. At elevated temperature metabolic activity of the organism is increased. Generally, temperatures variations do not have any noticeable effects on the various water uses of the river Bhagirathi from Gangotri to Rishikesh.

The analysis shows that the alkaline nature of water. The $\mathrm{pH}$ value of water increases from Gangotri to Rishikesh in summer and monsoon season this is due to the presence of sufficient quantities of carbonates in the river water while the alkaline nature of river water decreases from Rishikesh to Gangotri in the winter season (see point 3 in Tables 1-15). The fluctuations in $\mathrm{pH}$ can be due to exposes of river water to the atmosphere, some biological activities, and temperature changes. The calculated increases value of $\mathrm{pH}$ varies from summer to monsoon in all five station in three consecutive years (2013-2016) due to varied and uncertain nature of pollutants, falling in the river due to surface washing, falling of excess rain, Photosynthetic activity is also responsible for $\mathrm{pH}$ changes. Seasonal Variation in Mean Values of Physico-Chemical Parameters conductivity and Turbidity of River Bhagirathi at all five Sampling Station (2013-2016). Conductivity is increased from Gangotri to Rishikesh when we move from 
RASĀYAN $J$. Chem.

Vol. 10 | No. 4 |1167-1183 | October - December | 2017

summer to monsoon season (see point 4 in Tables 1-15) and decreases when go to the winter season in all three consecutive years 20013 to 2016. The conductivity is generally quite high from Uttarkashi to Rishikesh in summer and monsoon season indicates the presence of dissolved salts in water, making the taste sour and less suitable for drinking purpose. As the observation also shows, the conductivity is minimum in the winter season in Gangotri to Uttarkashi $(0.21$ to $0.22 \mu)$ (see point 4 , Tables $1,2,3)$ this may be attributed to the lower solubility of mineral salts at more alow temperature in river water and most suitable for potable.Rishikesh (13.93 NTU) has most turbid water which is likely to be unfit for domestic purpose, food and beverage industries and requires the treatment.In monsoon season the alkalinity of river water is quite high in sample station of Tehri and Rishikesh i.e. $211.56 \mathrm{ppm}$ which indicates that the suitability of water for irrigation and raw domestics uses.The observation is showing that the water quality of river Bhagirathi is quite good and amount of dissolved oxygen is maximum present in all season. The observed value of calcium is slightly higher as compared to other sample station from summer to monsoon in three consecutive years Rishikesh (13.54-25.80 ppm) (see point14 in Tables $5,10,15)$ due to the human activity and industrial pollution. Chloride content is the maximum in Rishikesh as compared to other sample stations due to human activity. The average value of sulfate in sample station Rishikesh (19.01ppm) (see point 17, Tables-5,10,15) is maximum. The average values of sodium in monsoon season slightly increase from Gangotri to Rishikesh of Bhagirathi river water due to weathering of various rocks, domestics rocks, flood etc.In the study of heavy metals like $\mathrm{Zn}, \mathrm{Pb}, \mathrm{Cr}, \mathrm{As}, \mathrm{Mn}, \mathrm{Ni}$ etc. are not-detectable because of these values in very low quantity and can't be analyzed. The river water of Bhagirathi is free from heavy metals and not causes any serious effects on health.

Table-1: Seasonal Variation of Physicochemical Parameters of River Bhagirathi at sampling station I-Gangotri (2013-2014).

\begin{tabular}{c|c|c|c|c}
\hline \multirow{2}{*}{ S.No. } & Physicochemical Parameters & Summer & Monsoon & Winter \\
\cline { 3 - 5 } & & (Mar-Jun) & (Jul-Oct) & (Nov-Feb) \\
\hline 1 & Colour & Colourless & Colourless & Colourless \\
\hline 3 & Temperature $\left({ }^{0} \mathrm{C}\right)$ & 18.80 & 15.31 & 8.62 \\
\hline 4 & $\mathrm{pH}$ & 7.19 & 7.31 & 7.05 \\
\hline 5 & Conductivity $(\mu \mathrm{S} / \mathrm{cm})$ & 0.23 & 0.28 & 0.21 \\
\hline 6 & Turbidity $(\mathrm{NTU})$ & 5 & 9.9 & 5.1 \\
\hline 7 & Alkalinity $(\mathrm{ppm})$ & 150.31 & 162.31 & 122.52 \\
\hline 8 & Total solids $(\mathrm{ppm})$ & 89.31 & 116.51 & 65.29 \\
\hline 9 & Total Dissolved solids $(\mathrm{ppm})$ & 74.3 & 92.21 & 49.11 \\
\hline 10 & Total Suspended Solids $(\mathrm{ppm})$ & 15.05 & 23.24 & 16.2 \\
\hline 11 & Dissolve Oxygen $(\mathrm{ppm})$ & 7.92 & 6.71 & 9.93 \\
\hline 12 & BOD (ppm) & 1.11 & 1.43 & 0.90 \\
\hline 13 & COD $(\mathrm{ppm})$ & 5.03 & 6.12 & 4.55 \\
\hline 14 & Total Hardness $(\mathrm{ppm})$ & 50.14 & 71.32 & 49.32 \\
\hline 15 & Calcium & 11.85 & 12.01 & 10.99 \\
\hline 16 & Magnesium & 5.03 & 8.97 & 6.11 \\
\hline 17 & Chloride & 4.12 & 8.25 & 3 \\
\hline 18 & Sulphate & 11.31 & 16 & 11.11 \\
\hline 19 & Sodium & 7.21 & 15.01 & 7.35 \\
\hline
\end{tabular}


RASĀYAN J. Chem.

Vol. 10 | No. 4 |1167-1183 | October - December | 2017

\begin{tabular}{c|c|c|c|c}
\hline 20 & Iron & 0.015 & 0.022 & 0.01 \\
\hline 21 & Zinc & Not Detected & Not Detected & Not Detected \\
\hline 22 & Copper & Not Detected & Not Detected & Not Detected \\
\hline 23 & Cadmium & Not Detected & Not Detected & Not Detected \\
\hline 24 & Mercury & Not Detected & Not Detected & Not Detected \\
\hline 25 & Chromium & Not Detected & Not Detected & Not Detected \\
\hline 26 & Lead & Not Detected & Not Detected & Not Detected \\
\hline 27 & Manganese & Not Detected & Not Detected & Not Detected \\
\hline 28 & Nickel & Not Detected & Not Detected & Not Detected \\
\hline 29 & Arsenic & Not Detected & Not Detected & Not Detected \\
\hline
\end{tabular}

Table-2: Seasonal Variation of Physico-Chemical Parameters of River Bhagirathi at sampling station II - Harshil

\begin{tabular}{|c|c|c|c|c|}
\hline \multirow[t]{2}{*}{ S.No. } & \multirow[t]{2}{*}{ Physicochemical Parameters } & Summer & Monsoon & Winter \\
\hline & & (Mar-Jun) & (Jul-Oct) & (Nov-Feb) \\
\hline 1 & Colour & Colourless & Colourless & Colourless \\
\hline 2 & Temperature $\left({ }^{0} \mathrm{C}\right)$ & 19.35 & 15.62 & 8.9 \\
\hline 3 & $\mathrm{pH}$ & 7.22 & 7.4 & 7.1 \\
\hline 4 & Conductivity $(\mu \mathrm{S} / \mathrm{cm})$ & 0.23 & 0.28 & 0.21 \\
\hline 5 & Turbidity (NTU) & 5.52 & 8.92 & 6.31 \\
\hline 6 & Alkalinity(ppm) & 152.31 & 176.31 & 135.51 \\
\hline 7 & Total solids (ppm) & 90.24 & 120.31 & 64.1 \\
\hline 8 & Total Dissolved solids (ppm) & 74.32 & 87.2 & 51.3 \\
\hline 9 & Total Suspended Solids (ppm) & 15.93 & 28.11 & 14.8 \\
\hline 10 & Dissolve Oxygen (ppm) & 6 & 8.62 & 10.51 \\
\hline 11 & BOD (ppm) & 2 & 2.33 & 1 \\
\hline 12 & COD (ppm) & 5.15 & 6.2 & 5 \\
\hline 13 & Total Hardness (ppm) & 52.23 & 73.23 & 49.91 \\
\hline 14 & Calcium & 11.21 & 14.21 & 11.92 \\
\hline 15 & Magnesium & 6.21 & 10.2 & 7.8 \\
\hline 16 & Chloride & 4.77 & 8.63 & 4.21 \\
\hline 17 & Sulphate & 10.33 & 16.26 & 12.21 \\
\hline 18 & Sodium & 8.31 & 16.21 & 7.41 \\
\hline 19 & Potassium & 2.53 & 4.21 & 2.31 \\
\hline 20 & Iron & 0.015 & 0.022 & 0.014 \\
\hline 21 & Zinc & Not Detected & Not Detected & Not Detected \\
\hline 22 & Copper & Not Detected & Not Detected & Not Detected \\
\hline 23 & Cadmium & Not Detected & Not Detected & Not Detected \\
\hline 24 & Mercury & Not Detected & Not Detected & Not Detected \\
\hline
\end{tabular}


RASĀYAN J. Chem.

Vol. 10 | No. 4 |1167-1183 | October - December | 2017

\begin{tabular}{c|c|c|c|c}
\hline 25 & Chromium & Not Detected & Not Detected & Not Detected \\
\hline 26 & Lead & Not Detected & Not Detected & Not Detected \\
\hline 27 & Manganese & Not Detected & Not Detected & Not Detected \\
\hline 28 & Nickel & Not Detected & Not Detected & Not Detected \\
\hline 29 & Arsenic & Not Detected & Not Detected & Not Detected \\
\hline
\end{tabular}

Table-3: Seasonal Variation of Physico-Chemical Parameters of River Bhagirathi at sampling station III -Uttarkashi (2013-2014).

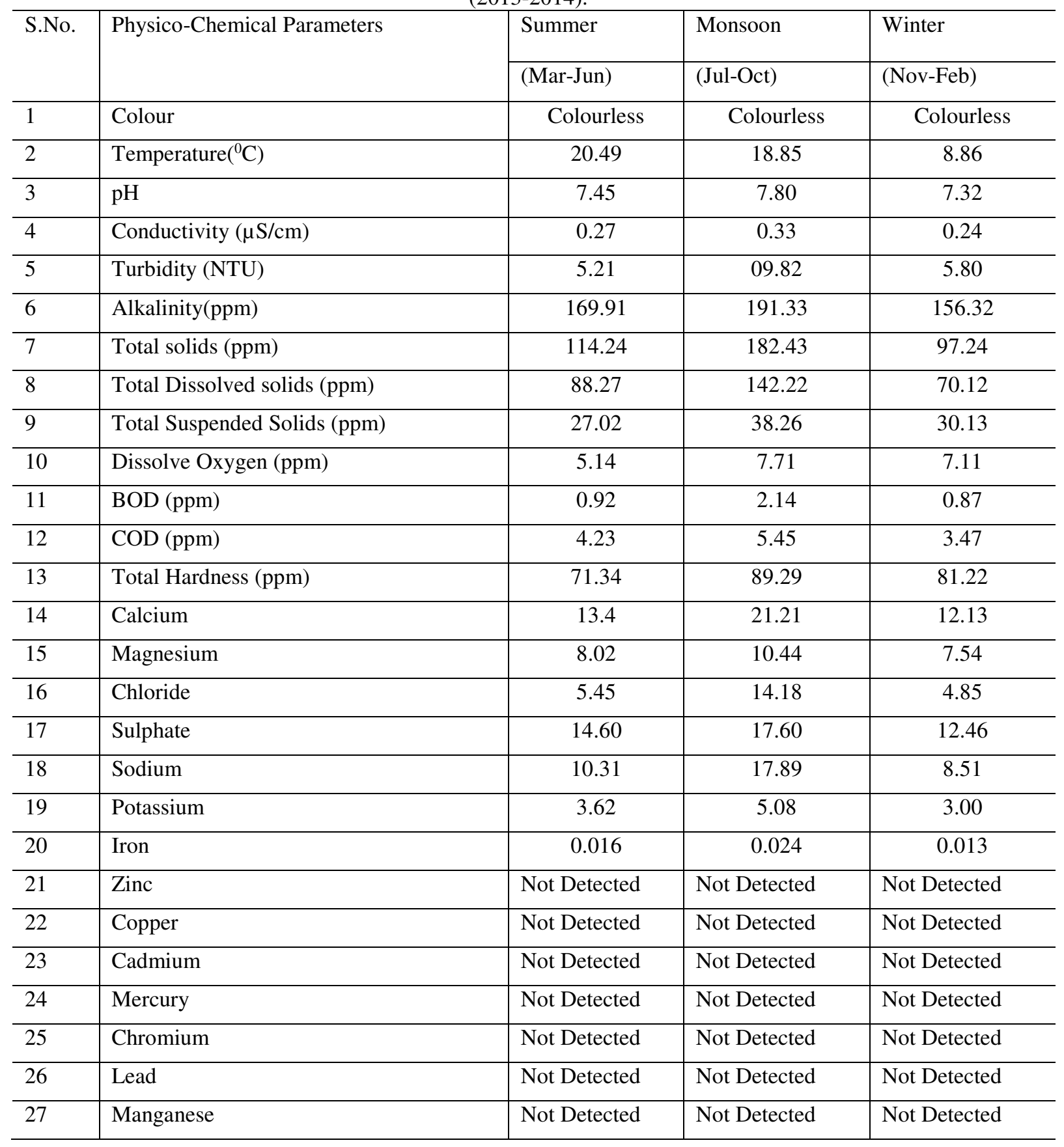


RASĀYAN J. Chem.

Vol. 10 | No. 4 |1167-1183 | October - December | 2017

\begin{tabular}{l|l|l|l|l}
\hline 28 & Nickel & Not Detected & Not Detected & Not Detected \\
\hline 29 & Arsenic & Not Detected & Not Detected & Not Detected \\
\hline
\end{tabular}

Table-4: Seasonal Variation of Physico-Chemical Parameters of River Bhagirathi at sampling station IV -Tehri

\begin{tabular}{|c|c|c|c|c|}
\hline & & 3-2014). & & \\
\hline S.No. & Physico-Chemical Parameters & Summer & Monsoon & Winter \\
\hline & & (Mar-Jun) & (Jul-Oct) & (Nov-Feb) \\
\hline 1 & Colour & Colourless & Colourless & Colourless \\
\hline 2 & Temperature $\left({ }^{0} \mathrm{C}\right)$ & 20.85 & 19.80 & 10.12 \\
\hline 3 & $\mathrm{pH}$ & 7.65 & 7.89 & 7.26 \\
\hline 4 & Conductivity $(\mu \mathrm{S} / \mathrm{cm})$ & 0.31 & 0.38 & 0.27 \\
\hline 5 & Turbidity (NTU) & 6.71 & 12.81 & 5.82 \\
\hline 6 & Alkalinity(ppm) & 197.51 & 216.36 & 179.51 \\
\hline 7 & Total solids (ppm) & 134.13 & 215.31 & 100.50 \\
\hline 8 & Total Dissolved solids (ppm) & 98.34 & 155.12 & 77.22 \\
\hline 9 & Total Suspended Solids (ppm) & 26.86 & 46.20 & 25.23 \\
\hline 10 & Dissolve Oxygen (ppm) & 7.92 & 6.71 & 9.93 \\
\hline 11 & BOD (ppm) & 1.11 & 1.43 & 0.90 \\
\hline 12 & COD (ppm) & 5.03 & 6.12 & 4.55 \\
\hline 13 & Total Hardness (ppm) & 52.23 & 73.23 & 49.91 \\
\hline 14 & Calcium & 11.21 & 14.21 & 11.92 \\
\hline 15 & Magnesium & 6.21 & 10.20 & 7.80 \\
\hline 16 & Chloride & 5.45 & 14.18 & 4.85 \\
\hline 17 & Sulphate & 14.60 & 17.85 & 12.46 \\
\hline 18 & Sodium & 09.31 & 18.89 & 10.51 \\
\hline 19 & Potassium & 3.62 & 5.08 & 3.00 \\
\hline 20 & Iron & 0.016 & 0.024 & 0.013 \\
\hline 21 & Zinc & Not Detected & Not Detected & Not Detected \\
\hline 22 & Copper & Not Detected & Not Detected & Not Detected \\
\hline 23 & Cadmium & Not Detected & Not Detected & Not Detected \\
\hline 24 & Mercury & Not Detected & Not Detected & Not Detected \\
\hline 25 & Chromium & Not Detected & Not Detected & Not Detected \\
\hline 26 & Lead & Not Detected & Not Detected & Not Detected \\
\hline 27 & Manganese & Not Detected & Not Detected & Not Detected \\
\hline 28 & Nickel & Not Detected & Not Detected & Not Detected \\
\hline 29 & Arsenic & Not Detected & Not Detected & Not Detected \\
\hline
\end{tabular}


RASĀYAN $J$. Chem.

Vol. 10 | No. 4 |1167-1183 | October - December | 2017

Table-5: Seasonal Variation of Physico-Chemical Parameters of River Bhagirathi at sampling station V - Rishikesh (2013-2014).

\begin{tabular}{|c|c|c|c|c|}
\hline \multirow[t]{2}{*}{ S.No. } & \multirow[t]{2}{*}{ Physico-Chemical Parameters } & Summer & Monsoon & Winter \\
\hline & & (Mar-Jun) & (Jul-Oct) & (Nov-Feb) \\
\hline 1 & Colour & Colourless & Colourless & Colourless \\
\hline 2 & Temperature $\left({ }^{0} \mathrm{C}\right)$ & 20.85 & 19.80 & 10.12 \\
\hline 3 & $\mathrm{pH}$ & 7.65 & 7.89 & 7.26 \\
\hline 4 & Conductivity $(\mu \mathrm{S} / \mathrm{cm})$ & 0.31 & 0.38 & 0.27 \\
\hline 5 & Turbidity (NTU) & 6.71 & 12.81 & 5.82 \\
\hline 6 & Alkalinity(ppm) & 197.51 & 216.36 & 179.51 \\
\hline 7 & Total solids (ppm) & 134.13 & 215.31 & 100.50 \\
\hline 8 & Total Dissolved solids (ppm) & 98.34 & 155.12 & 77.22 \\
\hline 9 & Total Suspended Solids (ppm) & 26.86 & 46.20 & 25.23 \\
\hline 10 & Dissolve Oxygen (ppm) & 3.42 & 5.70 & 7.95 \\
\hline 11 & BOD (ppm) & 0.87 & 2.61 & 0.79 \\
\hline 12 & COD (ppm) & 2.92 & 5.28 & 2.76 \\
\hline 13 & Total Hardness (ppm) & 84.52 & 100.20 & 93.00 \\
\hline 14 & Calcium & 13.21 & 24.31 & 12.31 \\
\hline 15 & Magnesium & 10.31 & 13.21 & 9.50 \\
\hline 16 & Chloride & 7.23 & 17.31 & 6.42 \\
\hline 17 & Sulphate & 16.31 & 18.91 & 15.31 \\
\hline 18 & Sodium & 11.61 & 19.02 & 9.92 \\
\hline 19 & Potassium & 4.21 & 6.36 & 4.16 \\
\hline 20 & Iron & 0.019 & 0.028 & 0.017 \\
\hline 21 & Zinc & Not Detected & Not Detected & Not Detected \\
\hline 22 & Copper & Not Detected & Not Detected & Not Detected \\
\hline 23 & Cadmium & Not Detected & Not Detected & Not Detected \\
\hline 24 & Mercury & Not Detected & Not Detected & Not Detected \\
\hline 25 & Chromium & Not Detected & Not Detected & Not Detected \\
\hline 26 & Lead & Not Detected & Not Detected & Not Detected \\
\hline 27 & Manganese & Not Detected & Not Detected & Not Detected \\
\hline 28 & Nickel & Not Detected & Not Detected & Not Detected \\
\hline 29 & Arsenic & Not Detected & Not Detected & Not Detected \\
\hline
\end{tabular}

Table-6: Seasonal Variation of Physico-Chemical Parameters of River Bhagirathi at sampling station I- Gangotri (2014-2015).

\begin{tabular}{l|l|l|c|c}
\hline S.No. & Physico-Chemical Parameters & Summer & Monsoon & Winter \\
\cline { 3 - 5 } & & (Mar-Jun) & (Jul-Oct) & (Nov-Feb) \\
\hline 1 & Colour & Colourless & Colourless & Colourless \\
\hline
\end{tabular}


RASĀYAN J. Chem.

Vol. 10 | No. 4 |1167-1183 | October - December | 2017

\begin{tabular}{|c|c|c|c|c|}
\hline 2 & Temperature $\left({ }^{0} \mathrm{C}\right)$ & 19.45 & 14.57 & 8.29 \\
\hline 3 & $\mathrm{pH}$ & 7.22 & 7.4 & 7.1 \\
\hline 4 & Conductivity $(\mu \mathrm{S} / \mathrm{cm})$ & 0.21 & 0.3 & 0.2 \\
\hline 5 & Turbidity (NTU) & 5.39 & 8.79 & 5.59 \\
\hline 6 & Alkalinity(ppm) & 157.28 & 178.21 & 124.11 \\
\hline 7 & Total solids $(\mathrm{ppm})$ & 91.39 & 118.81 & 65.22 \\
\hline 8 & Total Dissolved solids (ppm) & 76.21 & 90.32 & 54.11 \\
\hline 9 & Total Suspended Solids (ppm) & 16.1 & 26.98 & 15.21 \\
\hline 10 & Dissolve Oxygen (ppm) & 8.86 & 8.82 & 9.86 \\
\hline 11 & BOD (ppm) & 0.98 & 1.48 & 0.88 \\
\hline 12 & COD (ppm) & 6.17 & 7.32 & 4.69 \\
\hline 13 & Total Hardness (ppm) & 53.1 & 74.28 & 50.23 \\
\hline 14 & Calcium & 11.31 & 14.41 & 10.80 \\
\hline 15 & Magnesium & 6.31 & 10.01 & 7.41 \\
\hline 16 & Chloride & 3.98 & 7.88 & 4.13 \\
\hline 17 & Sulphate & 11.31 & 17 & 11.29 \\
\hline 18 & Sodium & 8.1 & 14.65 & 7.25 \\
\hline 19 & Potassium & 2.61 & 4.12 & 2.21 \\
\hline 20 & Iron & 0.015 & 0.022 & 0.012 \\
\hline 21 & Zinc & Not Detected & Not Detected & Not Detected \\
\hline 22 & Copper & Not Detected & Not Detected & Not Detected \\
\hline 23 & Cadmium & Not Detected & Not Detected & Not Detected \\
\hline 24 & Mercury & Not Detected & Not Detected & Not Detected \\
\hline 25 & Chromium & Not Detected & Not Detected & Not Detected \\
\hline 26 & Lead & Not Detected & Not Detected & Not Detected \\
\hline 27 & Manganese & Not Detected & Not Detected & Not Detected \\
\hline 28 & Nickel & Not Detected & Not Detected & Not Detected \\
\hline 29 & Arsenic & Not Detected & Not Detected & Not Detected \\
\hline
\end{tabular}

Table-7: Seasonal Variation of Physico-Chemical Parameters of River Bhagirathi at sampling station II- Harshil

\begin{tabular}{c|c|c|c|c}
\hline \multicolumn{5}{c}{ (2014-2015). } \\
\cline { 3 - 5 } & Physico-Chemical Parameters & Summer & Monsoon & Winter \\
\cline { 3 - 5 } & & (Mar-Jun) & (Jul-Oct) & (Nov-Feb) \\
\hline 1 & Colour & Colourless & Colourless & Colourless \\
\hline 2 & Temperature $\left({ }^{0} \mathrm{C}\right)$ & 19.72 & 15.29 & 8.79 \\
\hline 3 & $\mathrm{pH}$ & 7.45 & 7.48 & 7.17 \\
\hline 5 & Conductivity $(\mu \mathrm{S} / \mathrm{cm})$ & 0.21 & 0.3 & 0.2 \\
\hline
\end{tabular}


RASĀYAN J. Chem.

Vol. 10 | No. 4 |1167-1183 | October - December | 2017

\begin{tabular}{|c|c|c|c|c|}
\hline 6 & Alkalinity(ppm) & 157.28 & 178.21 & 124.11 \\
\hline 7 & Total solids (ppm) & 90.31 & 119.31 & 70.31 \\
\hline 8 & Total Dissolved solids (ppm) & 76.21 & 90.32 & 54.11 \\
\hline 9 & Total Suspended Solids (ppm) & 16.1 & 26.98 & 15.21 \\
\hline 10 & Dissolve Oxygen (ppm) & 9.02 & 8.63 & 9.61 \\
\hline 11 & $\mathrm{BOD}(\mathrm{ppm})$ & 1.03 & 1.35 & 0.92 \\
\hline 12 & COD $(\mathrm{ppm})$ & 5.03 & 6.3 & 4.55 \\
\hline 13 & Total Hardness (ppm) & 53.1 & 74.28 & 50.23 \\
\hline 14 & Calcium & 11.31 & 14.41 & 10.8 \\
\hline 15 & Magnesium & 6.22 & 11.01 & 8.01 \\
\hline 16 & Chloride & 4.69 & 8.88 & 4.31 \\
\hline 17 & Sulphate & 9.85 & 16.89 & 12.36 \\
\hline 18 & Sodium & 8.32 & 16.81 & 7.31 \\
\hline 19 & Potassium & 2.61 & 3.78 & 2.34 \\
\hline 20 & Iron & 0.015 & 0.022 & 0.011 \\
\hline 21 & Zinc & Not & Not Detected & Not Detected \\
\hline 22 & Copper & Not & Not Detected & Not Detected \\
\hline 23 & Cadmium & Not & Not Detected & Not Detected \\
\hline 24 & Mercury & Not & Not Detected & Not Detected \\
\hline 25 & Chromium & Not & Not Detected & Not Detected \\
\hline 26 & Lead & Not & Not Detected & Not Detected \\
\hline 27 & Manganese & Not & Not Detected & Not Detected \\
\hline 28 & Nickel & Not & Not Detected & Not Detected \\
\hline 29 & Arsenic & Not & Not Detected & Not Detected \\
\hline
\end{tabular}

Table-8: Seasonal Variation of Physico-Chemical Parameters of River Bhagirathi at sampling station III- Uttarkashi (2014-2015).

\begin{tabular}{l|l|c|c|c}
\hline S.No. & Physico-Chemical Parameters & Summer & Monsoon & Winter \\
\cline { 3 - 5 } & & (Mar-Jun) & (Jul-Oct) & (Nov-Feb) \\
\hline 1 & Colour & Colourless & Colourless & Colourless \\
\hline 2 & Temperature $\left({ }^{0} \mathrm{C}\right)$ & 20.62 & 19.00 & 9.90 \\
\hline 3 & $\mathrm{pH}$ & 7.45 & 7.76 & 7.29 \\
\hline 4 & Conductivity $(\mu \mathrm{S} / \mathrm{cm})$ & 0.27 & 0.31 & 0.19 \\
\hline 5 & Turbidity $(\mathrm{NTU})$ & 5.91 & 11.68 & 6.45 \\
\hline 6 & Alkalinity $(\mathrm{ppm})$ & 169.90 & 200.32 & 160.01 \\
\hline 7 & Total solids $(\mathrm{ppm})$ & 120.00 & 199.16 & 98.81 \\
\hline 8 & Total Dissolved solids $(\mathrm{ppm})$ & 91.23 & 165.00 & 71.00 \\
\hline 9 & Total Suspended Solids $(\mathrm{ppm})$ & 38.77 & 51.16 & 35.80 \\
\hline
\end{tabular}


RASĀYAN J. Chem.

Vol. 10 | No. 4 |1167-1183 | October - December | 2017

\begin{tabular}{l|l|c|c|c}
\hline 10 & Dissolve Oxygen (ppm) & 6.32 & 7.78 & 6.20 \\
\hline 11 & BOD (ppm) & 0.94 & 1.24 & 0.84 \\
\hline 12 & COD $(\mathrm{ppm})$ & 4.28 & 5.81 & 3.87 \\
\hline 13 & Total Hardness (ppm) & 78.62 & 97.21 & 81.21 \\
\hline 14 & Calcium & 12.81 & 20.90 & 11.87 \\
\hline 15 & Magnesium & 8.21 & 12.11 & 7.88 \\
\hline 16 & Chloride & 5.55 & 14.28 & 4.99 \\
\hline 17 & Sulphate & 14.81 & 17.10 & 12.23 \\
\hline 18 & Sodium & 10.31 & 18.10 & 9.13 \\
\hline 19 & Potassium & 3.68 & 5.18 & 3.13 \\
\hline 20 & Iron & 0.016 & 0.025 & 0.013 \\
\hline 21 & Zinc & Not Detected & Not Detected & Not Detected \\
\hline 22 & Copper & Not Detected & Not Detected & Not Detected \\
\hline 23 & Cadmium & Not Detected & Not Detected & Not Detected \\
\hline 24 & Mercury & Not Detected & Not Detected & Not Detected \\
\hline 25 & Chromium & Not Detected & Not Detected & Not Detected \\
\hline 26 & Lead & Not Detected & Not Detected & Not Detected \\
\hline 27 & Manganese & Not Detected & Not Detected & Not Detected \\
\hline 28 & Nickel & Not Detected & Not Detected & Not Detected \\
\hline 29 & Arsenic & Not Detected & Not Detected & Not Detected \\
\hline
\end{tabular}

Table-9: Seasonal Variation of Physico-Chemical Parameters of River Bhagirathi at sampling station IV- Tehri

\begin{tabular}{l|l|c|c|c}
\hline S.No. & Physico-Chemical Parameters & Summer & Monsoon & Winter \\
\cline { 3 - 5 } & & (Mar-Jun) & (Jul-Oct) & (Nov-Feb) \\
\hline 1 & Colour & Colourless & Colourless & Colourless \\
\hline 2 & Temperature $\left({ }^{0} \mathrm{C}\right)$ & 20.70 & 19.70 & 10.17 \\
\hline 3 & $\mathrm{pH}$ & 7.59 & 7.84 & 7.36 \\
\hline 4 & Conductivity $(\mu \mathrm{S} / \mathrm{cm})$ & 0.35 & 0.40 & 0.28 \\
\hline 5 & Turbidity $(\mathrm{NTU})$ & 7.91 & 15.01 & 6.98 \\
\hline 6 & Alkalinity $(\mathrm{ppm})$ & 195.21 & 211.21 & 177.01 \\
\hline 7 & Total solids $(\mathrm{ppm})$ & 125.31 & 200.22 & 99.23 \\
\hline 8 & Total Dissolved solids $(\mathrm{ppm})$ & 97.11 & 155.82 & 74.89 \\
\hline 9 & Total Suspended Solids $(\mathrm{ppm})$ & 28.20 & 44.40 & 24.34 \\
\hline 10 & Dissolve Oxygen $(\mathrm{ppm})$ & 3.58 & 5.67 & 7.49 \\
\hline 11 & BOD $(\mathrm{ppm})$ & 0.86 & 1.51 & 0.76 \\
\hline 12 & COD $(\mathrm{ppm})$ & 2.99 & 4.35 & 2.78 \\
\hline 13 & Total Hardness $(\mathrm{ppm})$ & 52.82 & 71.19 & 48.24 \\
\hline
\end{tabular}


RASĀYAN J. Chem.

Vol. 10 | No. 4 |1167-1183 | October - December | 2017

\begin{tabular}{l|l|c|c|c}
\hline 14 & Calcium & 10.8 & 13.28 & 11.61 \\
\hline 15 & Magnesium & 6.31 & 10.01 & 7.41 \\
\hline 16 & Chloride & 3.98 & 7.88 & 4.13 \\
\hline 17 & Sulphate & 14.81 & 19.10 & 14.23 \\
\hline 18 & Sodium & 10.31 & 18.10 & 9.13 \\
\hline 19 & Potassium & 3.68 & 5.18 & 3.13 \\
\hline 20 & Iron & 0.016 & 0.025 & 0.013 \\
\hline 21 & Zinc & Not Detected & Not Detected & Not Detected \\
\hline 22 & Copper & Not Detected & Not Detected & Not Detected \\
\hline 23 & Cadmium & Not Detected & Not Detected & Not Detected \\
\hline 24 & Mercury & Not Detected & Not Detected & Not Detected \\
\hline 25 & Chromium & Not Detected & Not Detected & Not Detected \\
\hline 26 & Lead & Not Detected & Not Detected & Not Detected \\
\hline 27 & Manganese & Not Detected & Not Detected & Not Detected \\
\hline 28 & Nickel & Not Detected & Not Detected & Not Detected \\
\hline 29 & Arsenic & Not Detected & Not Detected & Not Detected \\
\hline
\end{tabular}

Table-10: Seasonal Variation of Physico-Chemical Parameters of River Bhagirathi at sampling station V- Rishikesh

\begin{tabular}{l|l|c|c|c}
\hline \multirow{2}{*}{ S.No. } & Physico-Chemical Parameters & Summer & Monsoon & Winter \\
\cline { 3 - 5 } & & $($ Mar-Jun $)$ & $($ Jul-Oct $)$ & (Nov-Feb) \\
\hline 1 & Colour & Colourless & Colourless & Colourless \\
\hline 2 & Temperature $\left({ }^{\circ} \mathrm{C}\right)$ & 20.70 & 19.70 & 10.17 \\
\hline 3 & $\mathrm{pH}$ & 7.59 & 7.84 & 7.36 \\
\hline 4 & Conductivity $(\mu \mathrm{S} / \mathrm{cm})$ & 0.35 & 0.40 & 0.28 \\
\hline 5 & Turbidity $(\mathrm{NTU})$ & 7.91 & 15.01 & 6.98 \\
\hline 6 & Alkalinity(ppm) & 195.21 & 211.21 & 177.01 \\
\hline 7 & Total solids $(\mathrm{ppm})$ & 125.31 & 200.22 & 99.23 \\
\hline 8 & Total Dissolved solids $(\mathrm{ppm})$ & 97.11 & 155.82 & 74.89 \\
\hline 9 & Total Suspended Solids $(\mathrm{ppm})$ & 28.20 & 44.40 & 24.34 \\
\hline 10 & Dissolve Oxygen $(\mathrm{ppm})$ & 3.58 & 5.67 & 7.49 \\
\hline 11 & BOD $(\mathrm{ppm})$ & 0.86 & 1.51 & 0.76 \\
\hline 12 & COD (ppm) & 2.99 & 4.35 & 2.78 \\
\hline 13 & Total Hardness (ppm) & 83.21 & 109.21 & 95.21 \\
\hline 14 & Calcium & 13.86 & 27.11 & 13.42 \\
\hline 15 & Magnesium & 10.98 & 12.68 & 9.88 \\
\hline 16 & Chloride & 7.32 & 18.31 & 6.20 \\
\hline 17 & Sulphate & 16.51 & 19.01 & 14.99 \\
\hline
\end{tabular}


RASĀYAN J. Chem.

Vol. 10 | No. 4 |1167-1183 | October - December | 2017

\begin{tabular}{l|l|c|c|c}
\hline 18 & Sodium & 11.31 & 18.91 & 9.31 \\
\hline 19 & Potassium & 4.33 & 6.69 & 3.92 \\
\hline 20 & Iron & 0.023 & 0.030 & 0.018 \\
\hline 21 & Zinc & Not Detected & Not Detected & Not Detected \\
\hline 22 & Copper & Not Detected & Not Detected & Not Detected \\
\hline 23 & Cadmium & Not Detected & Not Detected & Not Detected \\
\hline 24 & Mercury & Not Detected & Not Detected & Not Detected \\
\hline 25 & Chromium & Not Detected & Not Detected & Not Detected \\
\hline 26 & Lead & Not Detected & Not Detected & Not Detected \\
\hline 27 & Manganese & Not Detected & Not Detected & Not Detected \\
\hline 28 & Nickel & Not Detected & Not Detected & Not Detected \\
\hline 29 & Arsenic & Not Detected & Not Detected & Not Detected \\
\hline
\end{tabular}

Table-11: Seasonal Variation of Physico-Chemical Parameters of River Bhagirathi at sampling station I- Gangotri (2015-2016).

\begin{tabular}{l|l|c|c|c}
\hline \multirow{2}{*}{ S.No. } & Physico-Chemical Parameters & Summer & Monsoon & Winter \\
\cline { 2 - 4 } & & (Mar-Jun) & (Jul-Oct) & (Nov-Feb) \\
\hline 1 & Colour & Colourless & Colourless & Colourless \\
\hline 2 & Temperature $\left({ }^{0} \mathrm{C}\right)$ & 19.5 & 13.6 & 8.36 \\
\hline 3 & $\mathrm{pH}$ & 7.2 & 7.3 & 7.07 \\
\hline 4 & Conductivity $(\mu \mathrm{S} / \mathrm{cm})$ & 0.24 & 0.26 & 0.22 \\
\hline 5 & Turbidity $(\mathrm{NTU})$ & 5.1 & 10.21 & 5.3 \\
\hline 6 & Alkalinity(ppm) & 160.31 & 180.21 & 121.33 \\
\hline 7 & Total solids $(\mathrm{ppm})$ & 90 & 120.31 & 66.3 \\
\hline 8 & Total Dissolved solids $(\mathrm{ppm})$ & 75 & 95.3 & 50.2 \\
\hline 9 & Total Suspended Solids $(\mathrm{ppm})$ & 15 & 26 & 16.11 \\
\hline 10 & Dissolve Oxygen $(\mathrm{ppm})$ & 7.80 & 7.74 & 9.86 \\
\hline 11 & BOD $(\mathrm{ppm})$ & 1.15 & 1.46 & 0.95 \\
\hline 12 & COD $(\mathrm{ppm})$ & 5.22 & 6.17 & 4.63 \\
\hline 13 & Total Hardness $(\mathrm{ppm})$ & 53.31 & 75.21 & 49.12 \\
\hline 14 & Calcium & 11.8 & 13 & 11 \\
\hline 15 & Magnesium & 6.23 & 10.34 & 7.25 \\
\hline 16 & Chloride & 4.32 & 8.31 & 4.01 \\
\hline 17 & Sulphate & 10 & 15 & 11 \\
\hline 18 & Sodium & 8.25 & 16 & 7.6 \\
\hline 19 & Potassium & 2.55 & 4.3 & 2.02 \\
\hline 20 & Iron & 0.016 & 0.023 & 0.013 \\
\hline 21 & Zinc & Not Detected & Not Detected & Detected \\
\hline
\end{tabular}


RASĀYAN J. Chem.

Vol. 10 | No. 4 |1167-1183 | October - December | 2017

\begin{tabular}{l|l|l|l|l}
\hline 22 & Copper & Not Detected & Not Detected & Not Detected \\
\hline 23 & Cadmium & Not Detected & Not Detected & Not Detected \\
\hline 24 & Mercury & Not Detected & Not Detected & Not Detected \\
\hline 25 & Chromium & Not Detected & Not Detected & Not Detected \\
\hline 26 & Lead & Not Detected & Not Detected & Not Detected \\
\hline 27 & Manganese & Not Detected & Not Detected & Not Detected \\
\hline 28 & Nickel & Not Detected & Not Detected & Not Detected \\
\hline 29 & Arsenic & Not Detected & Not Detected & Not Detected \\
\hline
\end{tabular}

Table-12: Seasonal Variation of Physico-Chemical Parameters of River Bhagirathi at sampling station II- Harshil

\begin{tabular}{|c|c|c|c|c|}
\hline & & p-2016). & & \\
\hline S.No. & Physico-Chemical Parameters & Summer & Monsoon & Winter \\
\hline & & (Mar-Jun) & (Jul-Oct) & (Nov-Feb) \\
\hline 1 & Colour & Colourless & Colourless & Colourless \\
\hline 2 & Temperature $\left({ }^{0} \mathrm{C}\right)$ & 19.98 & 14.63 & 8.68 \\
\hline 3 & $\mathrm{pH}$ & 7.28 & 7.3 & 7.08 \\
\hline 4 & Conductivity $(\mu \mathrm{S} / \mathrm{cm})$ & 0.24 & 0.26 & 0.22 \\
\hline 5 & Turbidity (NTU) & 5.4 & 9.82 & 5.22 \\
\hline 6 & Alkalinity(ppm) & 155.31 & 167.81 & 127.21 \\
\hline 7 & Total solids (ppm) & 96.11 & 131.81 & 68.3 \\
\hline 8 & Total Dissolved solids (ppm) & 76.89 & 94.31 & 52.8 \\
\hline 9 & Total Suspended Solids (ppm) & 16.32 & 28.5 & 15.5 \\
\hline 10 & Dissolve Oxygen (ppm) & 8.13 & 7.81 & 9.78 \\
\hline 11 & BOD (ppm) & 1.01 & 1.35 & 0.92 \\
\hline 12 & COD (ppm) & 5.2 & 6.3 & 4.75 \\
\hline 13 & Total Hardness (ppm) & 55.51 & 73.84 & 51.17 \\
\hline 14 & Calcium & 11.3 & 14.81 & 11.72 \\
\hline 15 & Magnesium & 6.32 & 10.35 & 7.89 \\
\hline 16 & Chloride & 4.69 & 8.39 & 4.33 \\
\hline 17 & Sulphate & 10.43 & 16.23 & 12.22 \\
\hline 18 & Sodium & 8.32 & 16.22 & 7.32 \\
\hline 19 & Potassium & 2.58 & 4.31 & 2.28 \\
\hline 20 & Iron & 0.016 & 0.023 & 0.013 \\
\hline 21 & Zinc & Not Detected & Not Detected & Not Detected \\
\hline 22 & Copper & Not Detected & Not Detected & Not Detected \\
\hline 23 & Cadmium & Not Detected & Not Detected & Not Detected \\
\hline 24 & Mercury & Not Detected & Not Detected & Not Detected \\
\hline 25 & Chromium & Not Detected & Not Detected & Not Detected \\
\hline
\end{tabular}


RASĀYAN J. Chem.

Vol. 10 | No. 4 |1167-1183 | October - December | 2017

\begin{tabular}{l|l|l|l|l}
\hline 26 & Lead & Not Detected & Not Detected & Not Detected \\
\hline 27 & Manganese & Not Detected & Not Detected & Not Detected \\
\hline 28 & Nickel & Not Detected & Not Detected & Not Detected \\
\hline 29 & Arsenic & Not Detected & Not Detected & Not Detected \\
\hline
\end{tabular}

Table-13: Seasonal Variation of Physico-Chemical Parameters of River Bhagirathi at sampling station IIIUttarkashi (2015-2016).

\begin{tabular}{|c|c|c|c|c|}
\hline \multirow[t]{2}{*}{ S.No. } & \multirow[t]{2}{*}{ Physico-Chemical Parameters } & Summer & Monsoon & Winter \\
\hline & & (Mar-Jun) & (Jul-Oct) & (Nov-Feb) \\
\hline 1 & Colour & Colourless & Colourless & Colourless \\
\hline 2 & Temperature $\left({ }^{0} \mathrm{C}\right)$ & 20.71 & 18.31 & 9.98 \\
\hline 3 & $\mathrm{pH}$ & 7.44 & 7.79 & 7.39 \\
\hline 4 & Conductivity $(\mu \mathrm{S} / \mathrm{cm})$ & 0.29 & 0.33 & 0.24 \\
\hline 5 & Turbidity (NTU) & 5.81 & 11.66 & 5.59 \\
\hline 6 & Alkalinity(ppm) & 168.12 & 194.12 & 165.12 \\
\hline 7 & Total solids (ppm) & 121.31 & 182.23 & 101.01 \\
\hline 8 & Total Dissolved solids (ppm) & 91.01 & 148.01 & 72.21 \\
\hline 9 & Total Suspended Solids (ppm) & 30.31 & 34.12 & 27.80 \\
\hline 10 & Dissolve Oxygen (ppm) & 5.22 & 6.89 & 7.31 \\
\hline 11 & BOD (ppm) & 0.91 & 1.10 & 0.81 \\
\hline 12 & COD (ppm) & 3.31 & 4.72 & 2.92 \\
\hline 13 & Total Hardness (ppm) & 74.21 & 94.12 & 80.13 \\
\hline 14 & Calcium & 12.61 & 21.21 & 12.11 \\
\hline 15 & Magnesium & 8.32 & 10.88 & 7.76 \\
\hline 16 & Chloride & 5.61 & 14.82 & 5.01 \\
\hline 17 & Sulphate & 14.72 & 17.81 & 12.36 \\
\hline 18 & Sodium & 10.11 & 17.90 & 8.76 \\
\hline 19 & Potassium & 3.71 & 5.24 & 3.07 \\
\hline 20 & Iron & 0.018 & 0.024 & 0.015 \\
\hline 21 & Zinc & Not Detected & Not Detected & Not Detected \\
\hline 22 & Copper & Not Detected & Not Detected & Not Detected \\
\hline 23 & Cadmium & Not Detected & Not Detected & Not Detected \\
\hline 24 & Mercury & Not Detected & Not Detected & Not Detected \\
\hline 25 & Chromium & Not Detected & Not Detected & Not Detected \\
\hline 26 & Lead & Not Detected & Not Detected & Not Detected \\
\hline 27 & Manganese & Not Detected & Not Detected & Not Detected \\
\hline 28 & Nickel & Not Detected & Not Detected & Not Detected \\
\hline 29 & Arsenic & Not Detected & Not Detected & Not Detected \\
\hline
\end{tabular}


RASĀYAN $J$. Chem.

Vol. 10 | No. 4 |1167-1183 | October - December | 2017

Table-14: Seasonal Variation of Physico-Chemical Parameters of River Bhagirathi at sampling station IV- Tehri (2015-2016).

\begin{tabular}{|c|c|c|c|c|}
\hline \multirow[t]{2}{*}{ S.No. } & \multirow[t]{2}{*}{ Physico-Chemical Parameters } & \multirow{2}{*}{$\begin{array}{c}\text { Summer } \\
\text { (Mar-Jun) }\end{array}$} & \multirow{2}{*}{$\begin{array}{l}\text { Monsoon } \\
\text { (Jul-Oct) }\end{array}$} & \multirow{2}{*}{$\frac{\text { Winter }}{\text { (Nov-Feb) }}$} \\
\hline & & & & \\
\hline 1 & Colour & Colourless & Colourless & Colourless \\
\hline 2 & Temperature $\left({ }^{0} \mathrm{C}\right)$ & 20.75 & 19.92 & 11.23 \\
\hline 3 & $\mathrm{pH}$ & 7.63 & 7.72 & 7.38 \\
\hline 4 & Conductivity $(\mu \mathrm{S} / \mathrm{cm})$ & 0.36 & 0.41 & 0.26 \\
\hline 5 & Turbidity (NTU) & 7.81 & 13.98 & 6.84 \\
\hline 6 & Alkalinity(ppm) & 198.21 & 207.11 & 171.11 \\
\hline 7 & Total solids (ppm) & 126.87 & 198.98 & 98.36 \\
\hline 8 & Total Dissolved solids (ppm) & 99.21 & 151.03 & 74.02 \\
\hline 9 & Total Suspended Solids (ppm) & 27.66 & 47.85 & 24.24 \\
\hline 10 & Dissolve Oxygen (ppm) & 3.52 & 5.66 & 7.90 \\
\hline 11 & BOD (ppm) & 1.01 & 1.35 & 0.92 \\
\hline 12 & COD (ppm) & 5.20 & 6.30 & 4.75 \\
\hline 13 & Total Hardness (ppm) & 53.31 & 86.21 & 49.12 \\
\hline 14 & Calcium & 11.3 & 14.81 & 11.72 \\
\hline 15 & Magnesium & 6.32 & 10.35 & 7.89 \\
\hline 16 & Chloride & 3.98 & 7.88 & 4.13 \\
\hline 17 & Sulphate & 14.72 & 17.81 & 12.36 \\
\hline 18 & Sodium & 10.11 & 17.90 & 8.76 \\
\hline 19 & Potassium & 3.71 & 5.24 & 3.07 \\
\hline 20 & Iron & 0.018 & 0.024 & 0.015 \\
\hline 21 & Zinc & Not Detected & Not Detected & Not Detected \\
\hline 22 & Copper & Not Detected & Not Detected & Not Detected \\
\hline 23 & Cadmium & Not Detected & Not Detected & Not Detected \\
\hline 24 & Mercury & Not Detected & Not Detected & Not Detected \\
\hline 25 & Chromium & Not Detected & Not Detected & Not Detected \\
\hline 26 & Lead & Not Detected & Not Detected & Not Detected \\
\hline 27 & Manganese & Not Detected & Not Detected & Not Detected \\
\hline 28 & Nickel & Not Detected & Not Detected & Not Detected \\
\hline 29 & Arsenic & Not Detected & Not Detected & Not Detected \\
\hline
\end{tabular}

\section{CONCLUSION}

The study of Bhagirathi river in different five stations with three consecutive years revealed that the increasing urbanization and industrialization increase the water pollution due to the dumping of their effluents into the river. Proper management of wastes and effluent has led to improving the quality of the river, so the river water is potable and is posing no threats to the survival of aquatic flora and fauna.GIS 
RASĀYAN J. Chem.

Vol. 10 | No. 4 |1167-1183 | October - December | 2017

and RS technology have clearly expressed its use its understanding the aspects accountable for retaining the hydrological cycle, mainly the vegetal cover, surface water bodies, lithotypes, and landform.

Table-15: Seasonal Variation of Physico-Chemical Parameters of River Bhagirathi at sampling station V- Rishikesh (2015-2016).

\begin{tabular}{|c|c|c|c|c|}
\hline \multirow[t]{2}{*}{ S.No. } & \multirow[t]{2}{*}{ Physico-Chemical Parameters } & Summer & Monsoon & Winter \\
\hline & & (Mar-Jun) & (Jul-Oct) & (Nov-Feb) \\
\hline 1 & Colour & Colourless & Colourless & Colourless \\
\hline 2 & Temperature $\left({ }^{0} \mathrm{C}\right)$ & 20.75 & 19.92 & 11.23 \\
\hline 3 & $\mathrm{pH}$ & 7.63 & 7.72 & 7.38 \\
\hline 4 & Conductivity $(\mu \mathrm{S} / \mathrm{cm})$ & 0.36 & 0.41 & 0.26 \\
\hline 5 & Turbidity (NTU) & 7.81 & 13.98 & 6.84 \\
\hline 6 & Alkalinity(ppm) & 198.21 & 207.11 & 171.11 \\
\hline 7 & Total solids (ppm) & 126.87 & 198.98 & 98.36 \\
\hline 8 & Total Dissolved solids (ppm) & 99.21 & 151.03 & 74.02 \\
\hline 9 & Total Suspended Solids (ppm) & 27.66 & 47.85 & 24.24 \\
\hline 10 & Dissolve Oxygen (ppm) & 3.52 & 5.66 & 7.90 \\
\hline 11 & BOD (ppm) & 0.88 & 1.62 & 0.80 \\
\hline 12 & COD (ppm) & 2.88 & 4.17 & 2.74 \\
\hline 13 & Total Hardness (ppm) & 82.31 & 103.21 & 93.31 \\
\hline 14 & Calcium & 13.55 & 25.98 & 12.51 \\
\hline 15 & Magnesium & 10.78 & 13.78 & 9.71 \\
\hline 16 & Chloride & 7.42 & 17.68 & 6.10 \\
\hline 17 & Sulphate & 16.42 & 18.88 & 14.82 \\
\hline 18 & Sodium & 11.51 & 19.11 & 10.01 \\
\hline 19 & Potassium & 4.45 & 6.81 & 3.34 \\
\hline 20 & Iron & 0.022 & 0.030 & 0.011 \\
\hline 21 & Zinc & Not Detected & Not Detected & Not Detected \\
\hline 22 & Copper & Not Detected & Not Detected & Not Detected \\
\hline 23 & Cadmium & Not Detected & Not Detected & Not Detected \\
\hline 24 & Mercury & Not Detected & Not Detected & Not Detected \\
\hline 25 & Chromium & Not Detected & Not Detected & Not Detected \\
\hline 26 & Lead & Not Detected & Not Detected & Not Detected \\
\hline 27 & Manganese & Not Detected & Not Detected & Not Detected \\
\hline 28 & Nickel & Not Detected & Not Detected & Not Detected \\
\hline 29 & Arsenic & Not Detected & Not Detected & Not Detected \\
\hline
\end{tabular}


RASĀYAN J. Chem.

Vol. 10 | No. 4 |1167-1183 | October - December | 2017

\section{REFERENCES}

1. N. Semwal and Pratima Akolkar, Cibtech Journal of Bio-Protocols, 1(2), 1(2012).

2. Harish Chandra Joshi, I. P. Pandey, International Journal of Innovative Research in Science, Engineering and Technology, 4(10), 9670(2015).

3. M. M. Sarin, S. Krshnaswami, J. R. Trivedi and K. K. Sharma, J. Earth System Sci.,101, 89(1992).

4. A. Chauhan, and S. Singh, Report and Oeinion, 2(9), 53(2010).

5. A. Kumar and A. Dua ,Global Journal of Environmental Science, 8(1), 49(2009).

6. A. Ayoade Adedolapo and Naresh Aggarwal, Zoology and Ecology, 22(1), 72(2012).

7. APHA, A VVW A and WPCF. Standard method for Examination of water and waste.25th Edition, (2005).

8. R.K. Trivedi, Enviro Media, Karad, 245-249 (1986).

9. R.K. Trivediand P.K. Goyal, Enviro-Media Karad, 3-34 and 36-96 (1986)

10. P.K. Goel, New Age International Publishers,(1986).

11. J. Gaillardet, J. Viers, B. Dupre, Treatise on Geochemistry,5, 225(2003).

12. R.C. Sharma, O.P. Gusainand C.P. Juyal, Ecology of high altitude river Bhiangana of Garhwal Himalaya In: Trivedi, R.K., (ed) River Pollution in India, Ashish Publishing House, New Delhi, 1130 (1990).

13. M.I. Hammer and K.A. Mac.Kihan, Hydrology and Quality of Water resources. Wiley New York, (1986)

[RJC-1753/2017] 